\title{
KVG-Revision: Ärzte beider Basel gegen Zwang zu Netzwerken mit Budget-Mitverantwortung
}

\begin{abstract}
Sehr geehrter Herr Präsident
Sehr geehrte Mitglieder des Zentralvorstandes

Die Vorstände unserer beiden Ärztegesellschaften haben sich intensiv mit der KVG-Revisionsvorlage betreffend «Managed Care» auseinandergesetzt. In der aktuell vorliegenden Fassung ist diese Vorlage für uns nicht akzeptabel.

Wir sind nicht grundsätzlich gegen vertraglich geregelte Netzwerke (unter Einbezug von Grundversorgern und Spezialisten), aber gegen die - allein dem aktuellen politischen Zeitgeist huldigende, bloss vordergründig guttönende zwingend damit verbundene «Budget-Mitverantwortung» der behandelnden Ärztinnen und Ärzte.
\end{abstract}

Unsere Haltung begründen wir u.a. wie folgt:

- Eine «Budget-Mitverantwortung» steht mindestens in potentiellem Widerspruch zur ärztlichen Ethik, wie sie seit dem Altertum für unsere Berufsausübung allgemein als richtig anerkannt wird (salus aegroti - suprema lex = das Wohl des Kranken als oberstes Gesetz!). Wir haben dem Patienten jederzeit das medizinisch Gebotene angedeihen zu lassen, ohne Rücksicht auf den damit für uns (oder unser Netzwerk) verbundenen finanziellen Gewinn oder Verlust. Selbstverständlich bedeutet das nicht, dass wir neben der Zweckmässigkeit nicht auch die Wirtschaftlichkeit medizinischer Leistungen $\mathrm{zu}$ berücksichtigen haben. Es widerstrebt aber unserer ärztlichen Grundhaltung, ein Patientenkollektiv nach einem vorgegebenen Budget $\mathrm{zu}$ «bewirtschaften».

- Durch eine Budget-Mitverantwortung würden wir indirekt zu Mitvollstreckern rein wirtschaftlich motivierter Direktiven der Krankenversicherer gegenüber den Patienten. Dadurch würde zwangsläufig das Vertrauensverhältnis zwischen Patient und Ärztin bzw. Arzt beeinträchtigt, das eine wichtige Grundvoraussetzung für jede erfolgreiche Behandlung darstellt. Nicht zuletzt in diesem Sinne ist die infragestehende Budgetmitverantwortung ein völlig falscher Ansatz, um Gesundheitskosten zu sparen.

Medizinische Gesellschaft Basel Marktgasse 5

CH-4051 Basel

Tel. 0615601515

Fax 0615601516

Ärztegesellschaft Baselland

Renggenweg 1

CH-4450 Sissach

Tel. 0619769800

Fax 0619769801
Lebens stehen, bei Annahme der Vorlage noch erheblich zunehmen.

- Die Vorlage würde zweifellos die von der Schweizer Bevölkerung stets hochgehaltene Arzt- und Therapiewahlfreiheit empfindlich einschränken.

- Es ist inakzeptabel, dass ohne Not ein ParadigmenWechsel herbeigezwungen werden soll, der sich diesmal nicht nur zulasten der Ärzteschaft, sondern ebenso zulasten der Patienten auswirken würde. Ein derart einschneidendes Vorgehen stünde in krassem Missverhältnis zur fehlenden Evidenz einer immer wieder behaupteten Qualitätssteigerung (vielmehr ist das Gegenteil zu befürchten). Zudem wäre nicht einmal ein Kostenvorteil gesichert. (Auch die - auf rein theoretischen Annahmen basierenden - Zahlen der bisher vorliegenden Studien kommen auf eine Prämienverminderung in der Grundversicherung von weniger als 1\%).

- Wenn die Vertragsnetze bezüglich Qualität und Kosten derart effizient wären, wie zur Begründung der Vorlage behauptet wird, hätten sie sich in den rund 20 Jahren ihres Bestehens längst flächendeckend durchgesetzt.

Aus allen diesen Gründen besteht keinerlei Sachzwang, Vertragsnetze zum Quasi-Obligatorium zu erklären und dadurch die Patientenbedürfnisse zu missachten.

Wir halten es für verfehlt, wenn unsere zentrale Dachorganisation zu einer höchst fragwürdigen Vorlage hauptsächlich deswegen ja sagt, um dem Vorwurf der ewigen Neinsagerei zu entgehen. Wir sind es den Patienten und unseren Mitgliedern schuldig, auch gegen einen übermächtig erscheinenden politischen Zeitgeist konsequent die Interessen einer guten ärztlichen Berufsausübung zu vertreten. Dazu gehört die Ablehnung einer verpflichtenden ärztlichen Budgetmitverantwortung. Gegen medizinische Netzwerke (unter Einbezug aller Behandlungsstufen) und geeignetere Massnahmen zu deren Förderung haben wir nichts einzuwenden, im Gegenteil!

Für die Berücksichtigung unserer Stellungnahme danken wir Ihnen im Voraus bestens.

Mit freundlichen Grüssen

Medizinische Gesellschaft Basel,

Dr. Felix Eymann (Präsident),

Dr. Jennifer Langloh (Geschäftsführerin)

Ärztegesellschaft Baselland, Dr. Lukas Wagner (Präsident),

Friedrich Schwab (Sekretär) 